\title{
Use of Hydroxychloroquine in Hospitalized Patients With COVID-19 Pneumonia: Results of a Retrospective Study in a Community Hospital
}

\author{
Samantha Shojaie, DO ${ }^{1,2} \bullet$ Phi Le, D0 ${ }^{1,2} \bullet$ Martin Preciado, MD ${ }^{1,2} \bullet$ Harish Moorjani, MD ${ }^{1,3,4}$
}

S tudies have suggested in vitro activity of the antimalarial agent hydroxychloroquine (HCQ) against the SARS-CoV-2 virus, the causative agent of COVID-19. However, evidence regarding its in vivo efficacy in hospitalized patients with COVID19 pneumonia, representing a spectrum of moderate to severe to critical disease, remains limited. This retrospective study aimed to evaluate whether the use of HCQ in the treatment of hospitalized patients with COVID-19 pneumonia reduced mortality rates and rates of respiratory failure requiring intubation.

\section{METHODS}

From March 1 to March 31, 2020, 95 patients diagnosed with COVID-19 pneumonia were admitted to Phelps Hospi-

\section{AFFILIATIONS: \\ ${ }^{1}$ Phelps Hospital Northwell Health, Sleepy Hollow, New York ${ }^{2}$ Sleepy Hollow Open Door Family Medical Center, Sleepy Hollow, New York ${ }^{3}$ Hudson Infectious Diseases Associates, Briarcliff Manor, New York ${ }^{4}$ Westchester Medical Center, Valhalla, New York}

\section{CITATION:}

Shojaie S, Le P, Preciado M, Moorjani H. Use of hydroxychloroquine in hospitalized patients with COVID-19 pneumonia: results of a retrospective study in a community hospital. Consultant. Published online April 30, 2020. doi:10.25270/ con.2020.04.00016

Received April 21, 2020. Accepted April 27, 2020.

\section{DISCLOSURES:}

The authors report no relevant financial relationships.

\section{AUTHOR DECLARATIONS:}

All relevant ethical guidelines have been followed, and any necessary institutional review board (IRB) and/or ethics committee approvals have been obtained. All necessary patient/participant consent has been obtained, and the appropriate institutional forms have been archived.

\section{CORRESPONDENCE:}

Samantha Shojaie, DO, Phelps Hospital Northwell Health, 701 N Broadway, Ste 415, Sleepy Hollow, NY 10591 (sshojaie@northwell.edu) tal, a community hospital in Sleepy Hollow, Westchester County, New York. Among these patients, 81 received HCQ protocolized together with azithromycin, while 14 received no intervention. The 81 patients who received HCQ were divided into 3 groups: the early intervention group (within 0-5 days of symptom onset), the intermediate intervention group (within 6-10 days of symptom onset), and the late intervention group (greater than 10 days from symptom onset). The primary outcome assessed was the patient mortality rate. The secondary outcome assessed was the rate of intubation. QTc intervals, clinical data such as comorbidities, and initial laboratory values (white blood cell count, absolute neutrophil count, C-reactive protein [CRP] level, and lactate dehydrogenase $[\mathrm{LDH}]$ level) were recorded for each patient.

\section{RESULTS}

Of the 95 patients, 64\% (61) were men and 36\% (34) were women, with a median age of 61 years (range, 23 to 98 years). Of the 81 patients in the intervention group, the mortality rate was $22 \%$. The intubation rate in this group was $33 \%$ (27).

Of the patients started on HCQ within 0 to 5 days of symptom onset $(\mathrm{n}=44), 18(41 \%)$ were intubated during their admission, and 15 (34\%) died. Of the patients started on HCQ within 6 to 10 days of symptom onset $(n=29), 4(14 \%)$ were intubated, and $3(10 \%)$ died. Of patients started on HCQ more than 10 days after symptom onset $(n=8), 5(63 \%)$ were intubated, and none died. In comparison, the no-intervention group had a mortality rate of $14 \%$ (2 patients) and an intubation rate of $0 \%$.

Of the 81 patients in the intervention group, 69 had an electrocardiogram (ECG) during their course. Of those 69 patients, $35(51 \%)$ had prolonged QTc intervals, defined as greater than $440 \mathrm{~ms}$ in men and greater than $460 \mathrm{~ms}$ in women. Of the 14 patients in the no-intervention group, 10 had an ECG during their course; of those, $3(30 \%)$ had prolonged QTc intervals. 
In the intervention group, most patients were found to have 2 or more comorbid risk factors for severe illness, as well as abnormal elevations in initial CRP and LDH values. In contrast, patients in the no-intervention group had generally fewer comorbid risk factors and lower initial levels of laboratory biomarkers such as CRP and LDH.

\section{DISCUSSION}

No decrease in mortality or intubation rates were observed between early, intermediate, and late initiation of HCQ in patients hospitalized with COVID-19 pneumonia in this retrospective study. Findings in the no-intervention group also suggested no relative increase in rates of mortality and intubation. Initial laboratory values did not differ significantly within the intervention group but suggested an elevated inflammatory state in most of those patients. Inflammatory biomarkers were also initially elevated in the no-intervention group, although to a lesser degree than in the intervention group, which also exhibited a greater number of comorbid risk factors for severe illness than did the no-intervention group.

At the same time, prolonged QTc intervals placing patients at risk for cardiac arrhythmias were found in a significant proportion of the intervention group while the no-intervention group had proportionally fewer instances of prolonged QTc intervals. These findings suggest that for patients with COVID-19 pneumonia requiring hospitalization (representing moderate to severe to critical disease), early initiation of HCQ offers no decrease in mortality rates nor rates of intubation, and that no initiation of HCQ furthermore shows no worse relative outcomes. An additional risk of QTc prolongation was also seen in the intervention group.

Among the limitations of the study are the significantly smaller sample size of the no-intervention group. This data set was identified subsequent to the initial retrospective evaluation of outcomes in the intervention group and was included as a control; however, no blinding or placebo treatment was part of the protocol. Within our hospital, protocols were changing frequently due to emerging studies and data from other institutions; this in part explains why fewer patients did not initially receive HCQ. Furthermore, the no-intervention group had a lesser degree of biomarker elevations and fewer comorbidities than the intervention group, and lower rates of progression of mortality and morbidity may be related to better initial clinical status. Our results represent only one hospital's experience during the COVID-19 pandemic. Further clinical studies should be undertaken to assess the role of HCQ in prophylaxis or early outpatient initiation for mild COVID-19 infection without pneumonia requiring hospitalization. 